\title{
A CHARACTERIZATION OF INNER PRODUCT SPACES
}

DAVID ALBERT SENECHALLE

The well-known parallelogram law of Jordan and von Neumann [1] has been generalized in two different ways by M. M. Day [2] and E. R. Lorch [3], and both of these results are corollaries of the theorem to be proved here. Jordan and von Neumann established that in order for a normed linear space $S$ to be an inner product space, it is necessary and sufficient that the following condition (JN) be satisfied: $\|P+Q\|^{2}+\|P-Q\|^{2}=2\|P\|^{2}+2\|Q\|^{2}$ for every $P$ and $Q$ in $S$. Day observed that $(\mathrm{JN})$ could be restricted to points of norm one; i.e.,

Condition (D) $\|P+Q\|^{2}+\|P-Q\|^{2}=4$ for every $P$ and $Q$ on the unit sphere for $S .{ }^{1}$

Lorch's condition, instead of stipulating the specific functional relation ( $\mathrm{JN}$ ) between $\|P-Q\|$ and $\|P\|,\|Q\|$, and $\|P+Q\|$, requires simply that there exist some functional relation between them.

Condition (L) There exists some function $F$ of three real variables such that $F(\|P\|,\|Q\|,\|P+Q\|)=\|P-Q\|$ for every $P$ and $Q$ in $S$.

The new condition $(\mathrm{S})$ is $(\mathrm{L})$ with the points restricted to the unit sphere: there exists some function $F$ of one real variable such that $F(\|P+Q\|)=\|P-Q\|$ for every $P$ and $Q$ of norm one. It is interesting to note that $(\mathrm{S})$ improves on $(\mathrm{L})$ in the same way that $(\mathrm{D})$ improves on (JN) and that (S) improves on (I)) in the same way that (L) improves on $(\mathrm{JN})$.

THEOREM. Suppose that $S$ is a normed linear space. The following two statements are equivalent:

(1) $S$ is an inner product space, and

(2) There exists some real function $F$ of one real variable defined over the number interval $[0,2]$ such that if each of $P$ and $Q$ is a point of the unit sphere for $S$, then $F(\|P+Q\|)=\|P-Q\|$.

Proof. It is easily seen that statement (1) implies statement (2), for if $S$ is an inner product space and $P$ and $Q$ are two points of the unit sphere for $S$, then $\|P+Q\|=\left(4-\|P-Q\|^{2}\right)^{1 / 2}$. Suppose that statement (2) is true. It may be assumed that $S$ is two dimensional, for (JN) implies that a normed linear space is an inner product space if each two-dimensional subspace of it is an inner product space.

Presented to the Society, April 15, 1967; received by the editors May 9, 1967.

${ }^{1}$ Examination of Day's proof reveals that the relation " =" in (D) may be replaced

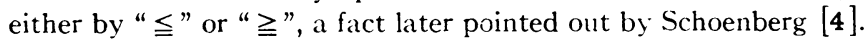


To begin the proof, denote by $M$ the unit sphere for $S$ and let $P$ be some point of $M$. There exists some point $Q$ of $M$ such that $\|P-Q\|$ $=1$. Set up a coordinate system with $P$ at $(1,0)$ and $Q$ at $(\cos (\pi / 3)$, $\sin (\pi / 3))$. Denote by $C$ the circle with radius 1 and center the origin. The argument that follows shows that $M$ is $C$ and therefore that $S$ is an inner product space.

The two points $P$ and $Q$ are common to $C$ and $M$. Since $|P-Q|$ $=\|P-Q\|=1, P-Q$ belongs to both $C$ and $M$. By the symmetry of $M, Q-P,-P$, and $-Q$ are also common to $C$ and $M$. Therefore,

$$
\begin{aligned}
\|P-Q\| & =\|Q-(Q-P)\|=\|Q-P-(-P)\|=\|-P-(-Q)\| \\
& =\|-Q-(P-Q)\|=\|(P-Q)-P\|=1 .
\end{aligned}
$$

Statement (2) implies that on the unit sphere, the norm of the sum (difference) of two points determines the norm of their difference (sum). Therefore,

$\|P+Q\|=\|2 Q-P\|=\|Q-2 P\|=\|-P-Q\|=\|P-2 Q\|=\|2 P-Q\|$.

Denote $\|P+Q\|$ by $c$. The two points $2 Q / c-P / c$ and $Q / c-2 P / c$ belong to the unit sphere. The norm of their difference is 1 and the norm of their sum is $3 / c$. This implies that $F(1)=3 / c$. But since $\|P-Q\|=1$ and $\|P+Q\|=c, F(1)=c$. This means that $c=3 / c$ and $c=(3)^{1 / 2}$. Since $|P+Q|=\|P+Q\|$, the point $(P+Q) /\|P+Q\|$ belongs to both $C$ and $M$. By a similar argument, $(2 Q-P) /\|2 Q-P\|$ belongs to both $C$ and $M$, and so forth, so that $\exp (2 \pi i n / 12)$ belongs to both $C$ and $M$ for $n=1,2,3, \cdots, 12$. This is presently to be extended to 24 points, 48 points, etc., but the method used on these first 12 points cannot be carried on.

The number interval $[0,2]$ is both the domain and the range of $F$. Also $F(0)=2, F(2)=0$, and $F(F(x))=x$ for each $x$ in $[0,2]$. Let $H$ be the transformation from the number interval $[0, \pi]$ to the set $F$ such that

$$
\begin{aligned}
& H(\theta)=\left(\left\|P-\frac{P \cos (\theta)+Q \sin (\theta)}{\|P \cos (\theta)+Q \sin (\theta)\|}\right\|,\right. \\
& \left.\left\|P+\frac{P \cos (\theta)+Q \sin (\theta)}{\|P \cos (\theta)+Q \sin (\theta)\|}\right\|\right) .
\end{aligned}
$$

The transformation $H$ is continuous, its domain is closed and compact, and its range is $F$. Therefore $F$ is a closed and compact point set, and it follows that $F$ is continuous. From this property it follows readily that $F$ is decreasing over $[0,2]$, for if $0 \leqq x<y \leqq 2$ and $F(x) \leqq F(y)$, continuity and $F(2)=0$ imply that there is a $y^{\prime}$ such that 
$x<y \leqq y^{\prime} \leqq 2$ and $F(x)=F\left(y^{\prime}\right)$, which is a contradiction since $F$ is its own inverse.

Suppose that $n$ is a positive integer and that the common part of $M$ and $C$ has as a subset some point set $K$ consisting of $6 \cdot 2^{n}$ points evenly spaced about $C$. It has been shown that this supposition is valid for $n=1$ and it will now be shown valid for $n+1$, implying that the common part of $M$ and $C$ is dense in $C$ and that $M$ is $C$. Suppose that $P_{0}, P_{1}, Q_{0}$, and $Q_{1}$ are four points of $K$ such that $P_{0}$ is adjacent to $P_{1}$ on $C, Q_{0}$ is adjacent to $Q_{1}$ on $C$, the point of $C$ midway between $P_{0}$ and $P_{1}$ on the shorter $\operatorname{arc}$ of $C$ from $P_{0}$ to $P_{1}$ is perpendicular to the point midway between $Q_{0}$ and $Q_{1}$ on the shorter arc of $C$ from $Q_{0}$ to $Q_{1}$ and $P_{1}$ is between $P_{0}$ and $Q_{1}$ on the shorter arc of $C$ from $P_{0}$ to $Q_{1}$. (That two points of $K$ are adjacent to each other on $C$ means that for some arc of $C$ containing the two points, no point of $K$ lies between them on that arc.) Rotate coordinates so that $(1,0)$ is halfway between $P_{0}$ and $P_{1}$ on the shorter arc of $C$. This puts both of $Q_{0}$ and $Q_{1}$ in either the upper half of the plane or in the lower half. The situation where $(0,1)$ is halfway between $Q_{0}$ and $Q_{1}$ on the shorter arc of $C$ is considered in the sequel and the other situation could be treated in a similar manner.

Let $P^{\prime}$ denote $\left(P_{0}+P_{1}\right) /\left\|P_{0}+P_{1}\right\|$, and $Q^{\prime},\left(Q_{0}+Q_{1}\right) /\left\|Q_{0}+Q_{1}\right\|$. It is first shown that $\left|P^{\prime}\right|=\left|Q^{\prime}\right|$. Let $m_{1}$ denote $\left|P^{\prime}\right|$ and let $m_{2}$ denote $\left|Q^{\prime}\right|$. Note that $\left\|P_{0}+P_{1}\right\|=\left|P_{0}+P_{1}\right| / m_{1}$ and that $\left\|Q_{0}+Q_{1}\right\|$ $=\left|Q_{0}+Q_{1}\right| / m_{2}$. Suppose that $m_{1} \neq m_{2}$. The case $m_{1}>m_{2}$ is considered and a contradiction obtained. The other case, $m_{2}>m_{1}$, would yield a contradiction in a similar manner.

Let $P_{0}^{\prime}$ denote the point of $K$ adjacent to $P_{0}$ and $C$ and such that $P_{0}$ is between $P_{0}^{\prime}$ and $P_{1}$ on $C$. Denote by $L$ the number $\left|P_{0}+P_{1}\right| / 2$. Because $M$ is the boundary of a convex point set, $|Z| \geqq L$ for each point $Z$ of $M$. Denote $\pi /\left(6 \cdot 2^{n}\right)$ by $\theta$, and note that $\theta$ is half the angle between any two adjacent members of $K$. Denote by $U$ the distance from the origin to the point on the $X$-axis that belongs to the line through $P_{0}$ and $P_{0}^{\prime}$. Because of convexity, $|Z| \leqq U$ for each point $Z$ of $M$. Let $A$ and $B$ denote $\left|P_{0}-P_{1}\right|$ and $\left|P_{0}+P_{1}\right|$ respectively. Let $Q_{2}$ be the member of $K$ adjacent to $Q_{1}$ on $C$ and such that $Q_{1}$ is between $Q_{0}$ and $Q_{2}$ on $C$. Denote by $s$ the slope of the line through $Q_{1}^{*}$ and $Q_{2}^{*}$ where "*" indicates complex conjugate. The following relationships may be verified by elementary calculations.

$$
\begin{aligned}
L & =\cos (\theta), \quad U=\cos (\theta) / \cos (2 \theta), \\
A & =\left|P_{0}-P_{1}\right|=2 \sin (\theta), \quad B=\left|P_{0}+P_{1}\right|=2 \cos (\theta), \\
\left|Q_{2}-Q_{2}^{*}\right| & =2 \cos (3 \theta), \quad s=\sin (2 \theta) / \cos (2 \theta), \quad Q_{1}=(A / 2, B / 2) .
\end{aligned}
$$


Since cosine is decreasing over $[0, \pi]$ and since $\theta$ and $3 \theta$ lie in this interval, $(\cos (\theta)+\cos (3 \theta)) / 2>\cos (3 \theta)$, so $\cos (\theta) \cos (2 \theta)>\cos (3 \theta)$, and $2 \cos (2 \theta)>2 \cos (3 \theta) / \cos (\theta)$. Now,

$$
\begin{aligned}
\left\|Q_{1}-Q_{1}^{*}\right\| & =2 \cos (\theta) / m_{2}>2 \cos (\theta) / m_{1}=\left\|P_{0}+P_{1}\right\| \\
& \geqq 2 \cos (\theta) / U=2 \cos (2 \theta)>2 \cos (3 \theta) / \cos (\theta) \\
& =\left|Q_{2}-Q_{2}^{*}\right| / L \geqq\left\|Q_{2}-Q_{2}^{*}\right\| .
\end{aligned}
$$

Therefore, $\left\|Q_{1}-Q_{1}^{*}\right\|>\left\|P_{0}+P_{1}\right\|>\left\|Q_{2}-Q_{2}^{*}\right\|$, implying that between the two vertical chords $Q_{1} Q_{1}^{*}$ and $Q_{2} Q_{2}^{*}$ of $M$, there is some vertical chord $R_{1} R_{2}$ of $M, R_{1}$ between $Q_{1}$ and $Q_{2}$ on $M$, such that $\left\|R_{1}-R_{2}\right\|=\left\|P_{0}+P_{1}\right\|$. By the hypothesis, $\left\|R_{1}+R_{2}\right\|=\left\|P_{0}-P_{1}\right\|$. The following equations may now be written.

$$
\begin{aligned}
& \frac{F\left(\left\|P_{0}+P_{1}\right\|\right)-F\left(\left\|Q_{0}+Q_{1}\right\|\right)}{\left\|Q_{0}+Q_{1}\right\|-\left\|P_{0}+P_{1}\right\|} \\
& =\frac{\left\|P_{0}-P_{1}\right\|-\left\|Q_{0}-Q_{1}\right\|}{\left\|Q_{0}+Q_{1}\right\|-\left\|P_{0}+P_{1}\right\|}=\frac{A / m_{2}-A / m_{1}}{B / m_{2}-B / m_{1}} \\
& =\frac{A}{B}=\frac{\left\|R_{1}+R_{2}\right\|-\left\|Q_{0}-Q_{1}\right\|}{\left\|Q_{0}+Q_{1}\right\|-\left\|R_{1}-R_{2}\right\|}=\frac{\left\|R_{1}+R_{2}\right\|-A / m_{1}}{B / m_{2}-\left|R_{1}-R_{2}\right| / m_{2}} .
\end{aligned}
$$

These equations are the beginning of a chain of inequalities which lead to a contradiction to the assumption that $m_{1} \neq m_{2}$.

Let $\left(x, r_{1}\right)$ be $R_{1}$ and let $\left(x,-r_{2}\right)$ be $R_{2}$ and suppose that $r_{1} \geqq r_{2}$. (The case $r_{2} \geqq r_{1}$ is just like this one and will not be considered.) Denote $r_{1}-r_{2}$ by $d$. The point $Q_{1}^{*}$ is $(A / 2,-B / 2)$ and the line through $Q_{1}^{*}$ and $Q_{2}^{*}$ has slope $s$. It is evident that $R_{2}$ cannot be above this line and that the maximum difference between $r_{1}$ and $r_{2}$, leaving $x$ fixed, occurs when $R_{1}$ and $R_{2}$ are each as high as possible. Since the line through $Q_{0}$ and $Q_{1}$ is parallel to the $X$-axis and since $R_{1}$ cannot be above this line, $r_{1} \leqq B / 2$. Similarly, $r_{2} \geqq B / 2-s(x-A / 2)$, so $d \leqq B / 2-(B / 2-s(x-A / 2))=s(x-A / 2)$. Let $r^{\prime}$ denote $B / 2$ $-s(x-A / 2)$. This gives the following.

$$
\begin{aligned}
\frac{A}{B} & =\frac{\left\|R_{1}+R_{2}\right\|-A / m_{1}}{B / m_{2}-\left|R_{1}-R_{2}\right| / m_{2}}=\frac{\left\|R_{1}+R_{2}\right\|-A / m_{1}}{B / m_{2}-\left(r_{1}+r_{2}\right) / m_{2}} \\
& \geqq \frac{\left\|R_{1}+R_{2}\right\|-A / m_{1}}{B / m_{2}-\left(2 r^{\prime}+d\right) / m_{2}} .
\end{aligned}
$$

Denote by $L_{1}$ the line through the origin and the point $R_{1}+R_{2}$. The slope $s_{1}$ of this line is $d /(2 x)$, and since $d \leqq s(x A / 2), s_{1} \leqq s(x-A / 2) /(2 x)$ 
$<s / 2<s$. This makes it clear that $L_{1}$ is not as steep as the line $L_{2}$ through $P_{0}$ and the point $\left(m_{1}, 0\right)$ so $L_{1}$ and $L_{2}$ have in common some point $Z$. If $L_{2}$ is vertical, then $m_{1}=L$ and $m_{2} \geqq m_{1}$, which is counter to the assumption made earlier that $m_{1}>m_{2}$. Therefore, $L_{2}$ is not vertical and has positive slope $s_{2}$. Note that $\| R_{1}+R_{2}|| \geqq\left|R_{1}+R_{2}\right| /|Z|$. Solving for $|Z|,|Z|=m_{1} s_{2}\left(4 x^{2}+d^{2}\right)^{1 / 2} /\left(2 s_{2} x-d\right)$, so $\left\|R_{1}+R_{2}\right\|$ $\geqq\left(2 s_{2} x-d\right) /\left(m_{1} s_{2}\right)$. Using this, the above inequality may be taken one step further.

$$
\frac{A}{B} \geqq \frac{\left\|R_{1}+R_{2}\right\|-A / m_{1}}{B / m_{2}-\left(2 r^{\prime}+d\right) / m_{2}} \geqq \frac{m_{2}\left(2 x-A-d / s_{2}\right)}{m_{1}(2 x-A) s} .
$$

Let $s_{2}^{\prime}$ denote the slope of the line through $P_{0}^{\prime}$ and $P_{0}$. It is evident that $s_{2}^{\prime} \leqq s_{2}$. Since $s_{2}^{\prime}=\cos (2 \theta) / \sin (2 \theta)$, it follows that $d / s_{2}$ $\leqq(x-A / 2) s / s_{2}^{\prime}=(x-A / 2) \sin ^{2}(2 \theta) / \cos ^{2}(2 \theta)<x-A / 2$. The chain of inequalities may now be terminated.

$$
\begin{aligned}
\frac{A}{B} & \geqq \frac{m_{2}\left(2 x-A-d / s_{2}\right)}{m_{1}(2 x-A) s} \geqq \frac{m_{2}}{2 m_{1} s} \geqq \frac{L}{2 U s} \\
& =\frac{\cos (\theta)}{2 \cos (\theta) \sin (2 \theta) / \cos ^{2}(2 \theta)}=\frac{\cos ^{2}(2 \theta)}{2 \sin (2 \theta)} .
\end{aligned}
$$

Since $A=2 \sin (\theta)$ and $B=2 \cos (\theta)$, $\sin (\theta) / \cos (\theta)>\cos ^{2}(2 \theta) / 2 \sin (2 \theta)$. By multiplying both sides by the product of the denominators and simplifying, one obtains $\cos (\theta)>5 \cos (3 \theta) / 2+\cos (5 \theta) / 2>5 \cos (3 \theta) / 2$ $\geqq 5 /\left(2(2)^{1 / 2}\right)>1$. This is a contradiction caused by the assumption that $m_{1} \neq m_{2}$ and proves that $m_{1}=m_{2}$.

For each in teger $k$, let $H(k)$ mean the point $\exp (i k \theta) /\|\exp (i k \theta)\|$ and let $A(k)$ denote $|H(k)|$. All the points $H(k)$ belong to $M$, and if $k$ is odd, $H(k)$ belongs to $K$ and hence to $C$. Using the results just obtained, it is shown below that $C$ contains all the points $H(k)$, implying that there exist $6 \cdot 2^{n+1}$ points of $M$ evenly spaced about $C$.

Since $\pi / 2=3 \cdot 2^{n} \theta$, the points $H(2 k)$ and $H\left(2 k+3 \cdot 2^{n}\right)$ are related to each other as are $P^{\prime}$ and $Q^{\prime}$ in the above, so for every integer $k, A(2 k)$ $=A\left(2 k+3 \cdot 2^{n}\right)$. If $k$ is an odd in teger, both $H(k)$ and $H\left(k+3 \cdot 2^{n}\right)$ belong to $K$, so $A(k)=A\left(k+3 \cdot 2^{n}\right)$. This means that $A(k)=A\left(k+3 \cdot 2^{n}\right)$ for every integer $k$. Suppose that $j$ is an integer, $0 \leqq j \leqq n$, and, for each integer $k, A(k)=A\left(k+3 \cdot 2^{n-j}\right)$. This supposition is valid for $j=0$. In case $j<n$, it may be shown by the following argument that $A(k)$ $=A\left(k+3 \cdot 2^{n-(j+1)}\right)$ for every integer $k$. Let $X, Y, U$, and $V$ be as follows: 


$$
\begin{aligned}
X & =\left\|H(k)+H\left(k+3 \cdot 2^{n-j}\right)\right\|=2 A(k) \cos \left(3 \cdot 2^{n-j-1} \theta\right) / A\left(k+3 \cdot 2^{n-j-1}\right) ; \\
Y & =\left\|H(k)-H\left(k+3 \cdot 2^{n-j}\right)\right\| \\
& =2 A(k) \sin \left(3 \cdot 2^{n-j-1} \theta\right) / A\left(k+3 \cdot 2^{n-j-1}+3 \cdot 2^{n}\right) \\
& =2 A(k) \sin \left(3 \cdot 2^{n-j-1} \theta\right) / A\left(k+3 \cdot 2^{n-j-1}\right) ; \\
U & =\left\|H\left(k+3 \cdot 2^{n-j-1}\right)+H\left(k+3 \cdot 2^{n-j-1}+3 \cdot 2^{n-j}\right)\right\| \\
& =2 A\left(k+3 \cdot 2^{n-j-1}\right) \cos \left(3 \cdot 2^{n-j-1} \theta\right) / A(k) ; \\
V & =\left\|H\left(k+3 \cdot 2^{n-j-1}\right)-H\left(k+3 \cdot 2^{n-j-1}+3 \cdot 2^{n-j}\right)\right\| \\
& =2 A\left(k+3 \cdot 2^{n-j-1}\right) \sin \left(3 \cdot 2^{n-j-1} \theta\right) / A(k) .
\end{aligned}
$$

Suppose that $X \neq U$. Then

$$
\frac{F(X)-F(U)}{X-U}=\frac{Y-V}{X-U}=\frac{\sin \left(3 \cdot 2^{n-j-1} \theta\right)}{\cos \left(3 \cdot 2^{n-j-1} \theta\right)}>0 .
$$

This contradicts the fact proved earlier that $F$ is decreasing. Therefore, $X=U$ and this implies that for each integer $k, A(k)$ $=A\left(k+3 \cdot 2^{n-(j+1)}\right)$. Therefore, by induction, $A(k)=A(k+3)$ for every integer $k$, and this puts all the points $H(k)$ on $C$. Thus $M$ contains $6 \cdot 2^{n+1}$ points evenly spaced about $C$.

It has now been shown that there exist $6 \cdot 2^{1}$ points of $M$ evenly spaced about $C$ and that if there exist $6 \cdot 2^{n}$ points of $M$ evenly spaced on $C$, then there exist $6 \cdot 2^{n+1}$ such points. Thus the common part of $M$ and $C$ is dense in $C$ and $M$ is $C$, completing the proof to the theorem.

This result is easily applied to a complex normed linear space. Associated with every complex normed linear space $S_{C}$, there is a real normed linear space $S_{R}$ which has the same points as $S_{C}$, the same point addition, and the same meaning for multiplication of points by real numbers as in $S_{C}$. It is easily shown that $S_{C}$ is a complex inner product space if and only if $S_{R}$ is a real inner product space. If in $S_{C}$ it is true that on the unit sphere the norm of the sum of two points determines the norm of their difference, then the same is true for $S_{R}$, implying that $S_{R}$ is a real inner product space and hence that $S_{C}$ is a complex inner product space.

\section{BIBLIOGRAPHY}

1. P. Jordan and J. von Neumann, On inner products in linear metric spaces, Ann. of Math. (2) 36 (1935), 719-732. 
2. M. M. Day, Some characterizations of inner product spaces, Trans. Amer. Math. Soc. 62 (1947), 320-337.

3. E. R. Lorch, On certain implications which characterize Hilbert space, Ann. of Math. (2) 49 (1948), 523-532.

4. I. J. Schoenberg, A remark on M. M. Day's characterization of inner product spaces and a conjecture of L. M. Blumenthal, Proc. Amer. Math. Soc. 3 (1952), 961964.

The University of Texas and

Tracor, Inc., Austin, Texas 